Interactive comment on "The GGCMI Phase II

experiment: global gridded crop model simulations under uniform changes in $\mathrm{CO}_{2}$, temperature, water, and nitrogen levels (protocol version 1.0)" by James Franke et al.

James Franke et al.

jfranke@uchicago.edu

Received and published: 15 February 2020

Original comments in gray

Author responses in black 


\section{Anonymous Referee 1}

COMMENT: The manuscript by Franke et al., details the experimental design for the Phase II GGCMI crop model comparison. The goal is to provide a set of simulations to synchronize a variety of crop models and compare the responses from perturbations of temperature, precipitation, CO2, and nitrogen fertilizer. The result is a dataset of thousands of simulations that can be used to emulate statistical crop model response under varying inputs of climate change. The authors provide some analysis of the dataset, providing examples of non-linear behavior under multiple variable perturbations be-tween temperature, precipitation, and CO2. Furthermore, the authors provide access to other users for additional analysis. The manuscript is well written, the message is clearly defined, with a logical flow throughout, and void of technical errors. The authors did a good job detailing some of the more complex features of their study.

RESPONSE: Thank you for the overall assessment.

COMMENT: My main concern with this manuscript is I find the approach toward the perturbation experiments somewhat unrealistic. I understand the difficulty in generating simulations across different models in a way that is uniform, and I find the large number of simulations included in the dataset impressive but having such a large set of parameters for the simulations makes interpreting the output difficult and negates the heterogeneous(in space and time) behavior of climate. Wouldn't it be easier to use CMIP output to drive simulations which could reduce the number of model runs? Perhaps the authors could provide more discussion on this choice. Also, since these are offline runs, they don't include feedbacks between the atmosphere and land (e.g. irrigation feedbacks to temperature), which are important.

RESPONSE: Yes, the approach of using uniformly perturbed climate inputs does not reflect realistic climate scenarios. If the goal of GGCMI Phase II were to use these simulations for climate change impact assessments, this experimental design would be the wrong choice. However, the goals of GGCMI-II are to (i) scrutinize model response in

Printer-friendly version

Discussion paper
Interactive comment 
response to individual and combined drivers and to (ii) develop crop model emulators on these experiments. Both of those goals require sampling across the space of potential perturbations that allows untangling the contributions of individual factors that are highly correlated in realistic future scenarios (e.g. CO2 and temperature). That is, meeting our goals requires a suite of unrealistic inputs.

We do believe that GGCMI-II can also serve the needs of impacts assessments through the development of emulators. That is, the responses to the CTWN-A factors diagnosed from GGCMI-II can be used to build up emulations of what the crop models would produce under a realistic climate scenario, including all the heterogeneous aspects of true climate change. (In this exercise, a crop model emulator for each individual grid cell is driven with the timeseries of projected climate changes for that particular location.)

This use is not demonstrated in the manuscript here, which is the "experiment description" paper; instead it is shown in a companion "model description" paper that describes the CTWN-A emulators, and is now available as a GMD discussion paper (https://www.geosci-model-dev-discuss.net/gmd-2019-365/ ). GMD had requested that we split our discussion of GGCMI-II into these two components, to clearly distinguish the experimental description from the emulator development. In the companion paper we show that realistic CMIP-based simulations can be reproduced extremely well by emulators built from the CTWN-A experiment. This is true despite the fact that the uniform offset experiments omit some aspects of climate change that could be important to crops - the distribution of weather conditions within growing season, e.g. stronger warming in spring vs. summer. Such effects do not appear large enough to compromise the GGCMI-II emulators.

We have revised the text of the manuscript under review here to better explain the rationale of GGCMI-II and to point to the companion emulator paper as a justification of its utility for impacts assessment. This point is extremely important and we thank the reviewer for pointing out that our explanation was insufficiently clear in the submitted

Printer-friendly version

Discussion paper 
manuscript.

It is true that these experiments do not include feedbacks between irrigation and temperature. This is non-ideal, but in practice, the vast majority of crop yield projections under climate change also omit this feedback, and instead simply feed a climate projection to a process-based or statistical crop model. We have added some discussion of this point in the manuscript and suggested the need for future studies.

Interactive

COMMENT: I did not find the A1 simulations discussed anywhere. They seem to be included in the methods section but are not included in the analysis. Perhaps they should be omitted. Similarly, the nitrogen simulations are also missing from the analysis (except for the correlation with observations).

RESPONSE: The A dimension (adaptation in growing season length) is an integral part of the protocol and should be described fully in the experiment description paper. We have now given it more attention in the overall paper.

Note that the adaptation dimension will always be treated somewhat separately in discussion as it is not directly comparable to the other four dimensions (CTWN): it does not address inputs but the parameterization of crop varieties.

We have tried to limit the amount of analysis of results that are shown as experiment description papers are supposed to focus on experimental design, with a few results only as illustrations. However, we have added some additional material on $\mathrm{N}$ and $\mathrm{A}$ so that these dimensions do not seem less important.

\section{General Comments:}

COMMENT: P. 7, Section 2.3: The 12 models included in the study are very different types of models. I know this was discussed in the original paper describing protocol I, but it should also be noted here. How did the model differences inform the experimental design (or limit the scope of the study)?

Printer-friendly version

Discussion paper 
RESPONSE: Yes, the inclusion of different model types in a model intercomparison both complicates and enriches the analysis. One goal of the GGCMI Phase II experiment is to analyze model differences in order to better understand skills and deficiencies and to improve models. We have added this point more clearly in the text. Based on reviewer comments, we are also now adding a section describing key differences among models, and including a table in the supplemental material that describes model differences in inputs, structure and setup.

COMMENT: P. 9, L. 10: If some models don't output the anthesis date, why is it considered mandatory?

RESPONSE: The anthesis date is an important phenological indicator and was considered a standard output also in the previous stages of GGCMI. However, as some models do not explicitly compute anthesis dates, these cannot deliver these outputs. The "mandatory" label means that models that do compute anthesis should report it. We have modified the text and table caption to make this clear.

COMMENT: P. 15, L. 6: Is the negative impact on yield from increasing temperature due to shorter growing seasons or from actual heat damage to the crop?

RESPONSE: Typically, the effect is a combination of the two mechanisms. The use of both $\mathrm{A} 0$ and $\mathrm{A} 1$ setups was designed to answer exactly this question. We have added text to emphasize this point. Note however that the experiment description paper here is not intended to conduct all these analyses, but rather to describe the protocol and outputs of the experiments that will allow questions to be answered. We are glad that the experiment provokes such useful responses! GGCMI team members are currently preparing a paper describing in detail the effects of adaptation in these experiments.

COMMENT: P. 15, L 11-13: The change in yields at different latitudes is unrealistic because of the design of the experiment. Simply increasing temperature uniformly and not accounting for the seasonal differences in temperature change (i.e., stronger winter

Printer-friendly version

Discussion paper 
RESPONSE: As discussed above, the uniform perturbations are not intended to reproduce a realistic scenario and should not be used as such. The reviewer's comment is useful in telling us that we need to make this point more clear in the paper. We have added language to emphasize that the GGCMI-II output for a given uniform temperature shift should not be taken as a proxy for an actual projection under a realistic climate scenario that produces the equivalent global mean temperature change.

We have tried to clarify two important points brought up by this comment. First, the climate offsets in the GGCMI-II experiments refer to offsets during the growing season, not to annual means. The strong increases in yield in high-latitude regions in some simulations are therefore the appropriate response for each model given the applied level of warming during the growing season. Models of course disagree on the extent or even the sign of yield changes, especially in high-latitude regions, and their responses may be unrealistic.

Second, a scenario with a uniform offset (across space and time) will not match a scenario with the same mean change but with the spatial patterns of climate change expected under future scenarios. The effects of these spatial patterns are shown explicitly in the companion GMD "model description" paper (see link above). We now refer to that paper explicitly. 


\section{Anonymous Referee 2}

COMMENT: The manuscript by Franke et al. documented a new AgMIP GGCMI effort on simulating the crop responses to globally uniform environmental perturbations, including $\mathrm{CO} 2$, temperature, precipitation, nitrogen, and adaptation (CTWN-A). The simulation protocols are described in detail and key model outputs are made publically available. The authors made the first cut on data analysis to show the key characteristics of the simulated dataset. Overall, this manuscript is well organized and written. It also fulfills the scope of GMD and should be of great interests to the broader crop modeling and climate change adaptation community.

RESPONSE: Thank you for the overall positive assessment.

COMMENT: I have the following comments for the authors to consider:

COMMENT: Firstly, I see the nitrogen application rates designed in Table 1 are largely not realistic, especially considering how nitrogen application rates differ for different crops. I am not sure if I misunderstood anything there, but please help to clarify this point.

RESPONSE: The idea of the uniform perturbation and input levels in the CTWN-A experiment is not to be fully realistic but to allow for in-depth analyses by providing a structured analysis framework. Fertilizer application rates differ substantially across crops (e.g. maize vs. soybean) but also across the globe where access to fertilizers is often limited. We designed the ranges of CTWN so that low and high-end values are included and model behavior can be understood across these dimensions.

By using a range of nitrogen input levels (as well as inputs of climate variables), we are able to construct "emulators" of the crop model responses to these factors for arbitrary input levels. That is, the GGCMI-II experiments allow constructing a response surface that would allow reproducing the output crop models would have produced if run with more realistic (or indeed, any) nitrogen inputs. This use is explained in detail

Printer-friendly version

Discussion paper
Interactive comment 
in a companion GCM paper now available online at (https://www.geosci-model-devdiscuss.net/gmd-2019-365/ ). We have added more discussion in this first "experiment description" paper to make this clear, and now point to the companion paper.

COMMENT: Secondly, I found some critical information is missing in the current manuscript. For example, the differences among different models (especially those with the same base), the irrigation triggering rules in different models, key model inputs (such as cultivar in-formation), model tuning method and model spin-up design. Please see later detailed comments.

RESPONSE: We agree with this critique and have expanded the discussion of structural differences among models. We have also included a table in the Supplemental Materials showing key model features and structural differences. This addition will make the manuscript significantly more useful for readers.

COMMENT: Thirdly, there are 7 mandatory variables in Table 2. However, the authors only discussed yield, which I agree is the most important one for crop models. If the authors can have some discussions on other variables, it would be very interesting, even if the figures are dumped into supplementary materials.

RESPONSE: As this is the experiment description paper and the experiment is very comprehensive (experiments, different model types, different output variables), we tried to find a balance between producing a readable overview paper and one with exhaustive detail. However, we may have erred on the side of over-focusing on yield. We have therefore now added some examples of other outputs in the supplementary material and more discussion in the main text.

COMMENT: More detailed comments are as following: COMMENT: P2, L30-L31: the transition to "Global crop model experiments are needed for systematic climate change assessments" is a little wired to me. Are you talking about the same point with last sentence or not?

Discussion paper 
RESPONSE: Yes, this sentence is a bit too condensed. We have updated the language accordingly.

COMMENT: P3, L22: Folberth et al. (2016); Porwollik et al. (2017)-> Folberth et al. (2016) and Porwollik et al. (2017)?

RESPONSE: Thank you for catching this! These references are now corrected; we have updated Folberth et al. 2016 to Folberth et al. 2019.

COMMENT: P3, L25: (C3MP Ruane et al., 2014; McDermid et al., 2015)-> (C3MP) (Ruane et al.,2014; McDermid et al., 2015)

RESPONSE: We have changed to "(C3MP, see Ruane et al., 2014; McDermid et al., 2015)"

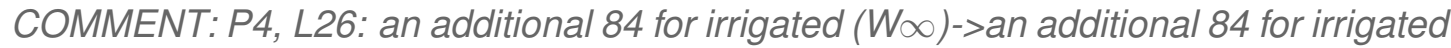
area $(W \infty)$ ? Are those 84 cases for irrigated area only with the assumption that the irrigated area will not change or also for rainfed area too (to get rid of water stress in rainfed regions)? Please clarify this point. It would be really interesting to have a no-water-limitation case for rainfed area. Moreover, how does each model trigger irrigation? Does the irrigated amount differ a lot among models?

RESPONSE: No, following the general GGCMI experiment design, fully rainfed and fully irrigated systems are simulated in all grid cells, independent of their actual distribution. This protocol allows for better analyses (e.g. simulations with and without water stress can be compared) and also for understanding the potential of optional cropland expansion. In the GGCMI Phase II setup, irrigated systems are also simulated during the rainfed growing seasons so that the simulation results are directly comparable and only differ with respect to water supply. We have modified the text to make this choice more clear.

COMMENT: Table 1: There are three levels of applied nitrogen (10, 60, $200 \mathrm{~kg} / \mathrm{ha}$ ). 
RESPONSE: Yes, as discussed above, $\mathrm{N}$ levels in the experimental protocol are uniformly applied across all locations and all crops, and are not intended to be realistic. The experiment design is intended to span the full range of plausible input values, though the maximum of $200 \mathrm{kgN} / \mathrm{ha}$ may actually be a bit low for some crops and regions. Using uniform offsets and input levels allows structured analysis of the effects of each factor. As per answers above, we have now made this rationale more clear in the text.

COMMENT: P7, L5: it would be good to document the main differences related with crop growth among those sharing-a-common-base models, i.e. EPIC group (EPICIIASA, EPIC-TAMU, GEPIC, pEPIC), and LPJ group (LPJMI, LPJ-GUESS).

RESPONSE: Yes, as discussed above, these differences are included in the table with details on model inputs, structure and setup that is now included in Supplementary Material. We feel this addition greatly strengthens the utility of the paper and thank the reviewer for the suggestion.

COMMENT: P7, L24-L25: will the change of phenological parameters have a huge impact on yield for different models?

RESPONSE: Yes, model performance can be very sensitive to the parametrization of growing seasons. That is why the experiment protocol prescribes harmonized growing seasons so that it is easier to analyze model responses. We have amplified discussion of this point in the text.

Printer-friendly version

Discussion paper

COMMENT: P7, L28: what's the "technical reasons" for CARAIB model? A note should be put on this.

RESPONSE: We agree that "technical reasons" does not adequately describe the is- 
sue at hand. In fact, the CARAIB team simply missed harmonizing this aspect. We think it is still of value to include their output in the archive, and any applications can exclude CARAIB results if required for their purposes. We have adjusted the sentence in question.

COMMENT: P7L35-P8L1: how did modelers adjust those parameters? Was it manual tuning or automatic tuning? And should this tuning be conducted for every year and each location? Ideally, there should be a section in the appendix for parameter tuning to include related details (parameter space, and tuning method)

RESPONSE: We now describe this procedure more clearly in the main text. The groups used manual parameter tuning to harmonize the growing seasons. First, parameters are adjusted for each crop in each location under the unperturbed AgMERRA baseline climate timeseries so that growing seasons in this 31-year period (1980-2010) reproduce specified observed average growing seasons for this period. For A0 simulations, the parameters are then left constant for all experiments, so that growing seasons alter under warming.

Note that because each crop model sets the growing season differently, the parameters modified will differ across models. Describing the exact procedure of the different modeling groups would require extensive discussion of the structure of each model. While we agree an appendix describing this would be useful to some readers, we feel it is out of scope for this paper. We hope that this need is satisfied instead by our links to the description papers for each individual model, which should cover their process of determining growing seasons.

COMMENT: P9, L10: please move "(Note that several models do not output the anthesis date.) "after "the dates of planting, anthesis, and maturity", i.e. the dates of planting, anthesis, and maturity (Note that several models do not output the anthesis date).

RESPONSE: Reviewer 1 also had problems with this sentence and it has been revised accordingly. It no longer includes parentheses.

Printer-friendly version

Discussion paper 
COMMENT: P9, L8: 30-year or 31-year (1980-2010)? What's the model spin-up protocol?

RESPONSE: The spin up is very different across models. This is now documented in the new table on model inputs, structure and setup.

COMMENT: P11, L20: no italic text in Table 3!

Interactive

comment

RESPONSE: Thank you for catching this. These simulation sets are shown in bold in Table 3 (column "Sims per crop"), and the sentence is now corrected.

COMMENT: P11, L28: did you missed 510 ppm there?

RESPONSE: Yes, thanks for catching this; it is now corrected.

COMMENT: P13, L26: For example, global correlation coefficients for maize in Phase I and Phase II are 0.89 and 0.74, respectively; for wheat 0.67 and 0.64, and for soybeans 0.64 and 0.59. (Compare to Müller et al. (2017) Figures 1-4 and 6.)-> For example, global correlation coefficients in Phase I and Phase II are 0.89 and 0.74 for maize, 0.67 and0.64 for wheat, and 0.64 and 0.59 for soybeans, respectively (Phase I values are from Figures 1-4 and 6 in Müller et al. (2017))

RESPONSE: Corrected as suggested.

COMMENT: P13, L27: Figure 2 should be Figure 2(c)-2(f)

RESPONSE: Corrected as suggested.

COMMENT: Caption of Figure 5: There are two "all" in "Figure shows all all simulated grid cells for each model"

Printer-friendly version

RESPONSE: Thanks for catching this; corrected.

COMMENT: P19, L1: region. (For soybeans, temperature effects are more complex; see Supple-mental Figure S5.)-> region (for soybeans, temperature effects are more 
COMMENT: P20, L10: Generally, the carbon fertilization effect (CFE) would be larger under drier condition than under wetter condition. Is this true in Fig. 6a and Fig. S7?? McGrath, J.M., Lobell, D.B. (2013). Regional disparities in the CO2 fertilization effect and implications for crop yields. Environmental Research Letters, 8, 014054

RESPONSE: The GGCMI-II experiment is designed to allow diagnosis of this and other interaction effects! But, as this is the experiment description paper, we are not analyzing results in full depth. We hope instead that many analyses will follow, making use of the freely available data set that we describe here. We have now added this citation and mentioned this effect as the possible target of a future study.

COMMENT: P21, L1-2: again, please check the use of parenthesis.

RESPONSE: We have removed parentheses here.

COMMENT: Section 5: I am glad that the authors discussed some of the limitations in the simulation exercise. One more point should be included there is about how to validate the simulated responses, especially considering that there are indeed some field experiments designed to measure the responses of crops to environmental manipulations.

RESPONSE: We now include more discussion of the fact that models have been individually and jointly evaluated, including against data from field experiments.

We also discuss the challenges from the artificial model setup in the GGCMI Phase II experiment more thoroughly, and now refer to the companion paper (Franke et al., 2020), in which we demonstrate that emulators built from this artificial setup can very well reproduce model behavior from crop yield simulations driven by more realistic future climate projections.

Printer-friendly version

Discussion paper 


\section{Reference:}

Franke J, Müller C, Elliott J, Ruane AC, Jägermeyr J, Snyder A, Dury M, Falloon P, Folberth C, François L, Hank T, Izaurralde RC, Jacquemin I, Jones C, Li M, Liu W, Olin S, Phillips M, Pugh TAM, Reddy A, Williams K, Wang Z, Zabel F, and Moyer E. 2020, The GGCMI phase II emulators: global gridded crop model responses to changes in CO2, temperature, water, and nitrogen (version 1.0), Geosci. Model Dev. Discuss., 2020, 1-31, doi: 10.5194/gmd-2019-365.

Interactive comment on Geosci. Model Dev. Discuss., https://doi.org/10.5194/gmd-2019-237, 2019. 